\title{
Una aproximación al estudio de los trabajos prácticos de laboratorio desde las representaciones de futuro personal docente de biología
}

\author{
An Approach to the Study of Laboratory Work From the Representations of Future \\ Biology Teachers
}

\section{Uma abordagem ao estudo dos trabalhos práticos de laboratório a partir das representações do futuro corpo docente de Biologia}

\author{
Erica Zorrilla \\ Universidad Nacional de San Juan \\ Consejo Nacional de Investigaciones Científicas y Técnicas \\ San Juan, Argentina \\ ericagabriela@gmail.com \\ https://orcid.org/0000-0001-6460-3319 \\ Claudia Mazzitelli \\ Universidad Nacional de San Juan \\ Consejo Nacional de Investigaciones Científicas y Técnicas \\ San Juan, Argentina \\ mazzitel@ffha.unsj.edu.ar \\ https://orcid.org/0000-0002-1199-4843
}



Recibido • Received • Recebido: 19 / 11 / 2019

Corregido • Revised • Revisado: 27 / 05 / 2021

Aceptado • Accepted • Aprovado: 05 / 07 / 2021

\section{Resumen:}

Introducción. En este artículo se presenta un estudio realizado con 111 estudiantes de la carrera de Formación Docente en Biología de la provincia de San Juan, Argentina, con el objetivo de conocer las opiniones de estudiantes, indagar sus representaciones sociales vinculadas con los trabajos prácticos de laboratorio y analizar su incidencia en el proceso actual de formación y en su futuro desempeño docente. Metodología. El estudio se desarrolló desde un enfoque cualitativo. Se implementó un instrumento que incluyó una técnica de evocación y jerarquización y una técnica de frases incompletas. Resultados. Los resultados obtenidos muestran que las representaciones tienden a centrarse en elementos vinculados a la enseñanza, al aprendizaje y a aspectos procedimentales. A su vez, las opiniones del estudiantado señalan la escasa realización de prácticas experimentales y la insuficiente vinculación con conceptos teóricos estudiados. Conclusión. Estos resultados invitan a reflexionar acerca de la necesidad de incrementar y diversificar el trabajo experimental en la formación docente de Biología para contribuir con el futuro desempeño profesional de estos estudiantes en el nivel secundario.

Palabras claves: Biología; formación docente; representaciones sociales; trabajos prácticos de laboratorio. 
http://doi.org/10.15359/ree.25-3.9

http://www.una.ac.cr/educare

educare@una.ac.cr

\begin{abstract}
:
Introduction. This article presents a study conducted with 111 students of the Biology teacher training program in San Juan Province, Argentina, to know the students' opinions, investigate their social representations related to the laboratory work, and analyze its impact on the current process and their future teaching performance. Method. The study was developed from a qualitative approach. The instrument implemented included a technique of evocation and hierarchical organization and a technique of incomplete phrases. Results. The results obtained show that the representations tend to focus on elements related to teaching, learning, and procedural aspects. At the same time, the opinions of the students indicate the scarce realization of experimental practices and the insufficient connection with the theoretical concepts studied. Conclusion. These results invite us to reflect on the need to increase and diversify experimental work in Biology teacher training to contribute to the future professional performance of these students at the secondary level.
\end{abstract}

Keywords: Biology; teacher training; social representations; laboratory work.

\begin{abstract}
Resumo:
Introdução. Este artigo apresenta um estudo realizado com 111 alunos da carreira de formação de professores de Biologia na província de San Juan, Argentina, com o objetivo de conhecer as opiniões dos alunos, investigar suas representações sociais relacionadas ao Trabalho Prático de Laboratório e analisar seu impacto no processo de treinamento atual e em seu desempenho de ensino futuro. Metodologia. O estudo foi desenvolvido a partir de uma abordagem qualitativa. Foi implementado um instrumento que incluiu uma técnica de evocação e classificação e uma técnica de frase incompleta. Resultados. Os resultados obtidos mostram que as representações tendem a enfocar elementos relacionados ao ensino, aprendizagem e aspectos procedimentais. Ao mesmo tempo, as opiniões dos alunos apontam para a escassa realização de práticas experimentais e a insuficiente ligação com os conceitos teóricos estudados. Conclusões. Estes resultados convidam-nos a reflectir sobre a necessidade de aumentar e diversificar o trabalho experimental na formação de professores de Biologia de forma a contribuir para o futuro desempenho profissional destes alunos do ensino secundário.
\end{abstract}

Palavras-chave: Biologia, formação de professores, representações sociais, trabalhos práticos de laboratório.

\title{
Introducción
}

La realización de trabajos prácticos de laboratorio (TPL) en el ámbito de la enseñanza de las ciencias ha sido cuestionada en numerosas ocasiones y por diversas causas (Fernández Marchesi et al., 2011; Hodson, 1994), atendiendo a que puede resultar en algunos casos particularmente compleja debido a que en su implementación interaccionan diferentes factores (institucionales, curriculares, personales, entre otros) (Mordeglia et al., 2006). Sin embargo, debido al carácter experimental de las ciencias naturales, no cabe duda de que los TPL deben ser un componente fundamental de los procesos de enseñanza y de aprendizaje de estas mismas (Fernández Marchesi y Amórtegui, 2017). 
http://doi.org/10.15359/ree.25-3.9

En este contexto se hace necesario ahondar en el pensamiento de estudiantes de carreras de formación docente en ciencias naturales, a fin de conocer sus opiniones sobre los TPL en general, y sobre las características de aquellos cursos prácticos que han realizado a lo largo de sus trayectorias educativas, y analizar en qué forma podrían incidir en el actual proceso de formación inicial y en su futuro desempeño docente. Un abordaje desde la teoría de las representaciones sociales (RS) resulta una opción válida por cuanto contribuye a abordar este estudio, en su complejidad, desde una perspectiva psicosocial que permite conocer la construcción e interpretación de la realidad por parte de los sujetos (Jodelet, 2003). De esta manera, el estudio de las RS sobre los TPL en el marco de la enseñanza y el aprendizaje de las ciencias naturales resulta un enfoque conceptual y metodológico útil, ya que permite identificar factores que obstaculizan y otros que benefician dichos procesos.

\section{Marco teórico}

La enseñanza de las ciencias naturales utiliza actividades y recursos didácticos comunes a otras disciplinas, como puede ser la búsqueda de información, la lectura de textos, el uso de recursos audiovisuales. No obstante, considerando el carácter experimental de estas ciencias, es necesario incorporar, en la propuesta de enseñanza, actividades específicas que favorezcan la interacción entre la teoría y la práctica para contribuir con el aprendizaje (Aguilar Muñoz et al., 2011), por ejemplo, a través de actividades relacionadas con el trabajo experimental, lo cual permite, entre otros aportes, poner en marcha mecanismos cognitivos necesarios para el aprendizaje del conocimiento científico y para la construcción de conceptos (Hernández-Millán et al., 2012).

Lo anteriormente mencionado pone en evidencia la importancia de realizar actividades experimentales en el aula de ciencias naturales. Sin embargo, para que estas actividades contribuyan con los procesos de enseñanza y de aprendizaje, deben ser organizadas, permitiendo contribuir con el logro de los objetivos propuestos. De esta manera los TPL deben articular diferentes tipos de actividades, mediante un enfoque integrado, en el que la teoría y la práctica se entrelazan en un tratamiento conjunto (del Carmen, 2011). Así, los TPL brindan, al estudiantado, la posibilidad de construir conocimiento a partir de sus propias experiencias, convirtiéndose en un importante recurso para el aprendizaje de muchos contenidos -tanto conceptuales, como procedimentales y actitudinales- (Merino y Herrero, 2007). También pueden y deben ser usados para estimular la curiosidad y el placer por la investigación y el descubrimiento, otorgando a cada estudiante la posibilidad de explorar, manipular, sugerir hipótesis, entre otras cuestiones (Bravo et al., 2016; Carrascosa et al., 2006; Gil, 1997), lo que permite el desarrollo de los procedimientos propios del quehacer científico. Además, en relación con la formación docente, constituyen un recurso didáctico que influye en el proceso de formación inicial y en el futuro desempeño profesional (De Pro Bueno, 1998, 1999). 
http://doi.org/10.15359/ree.25-3.9

http://www.una.ac.cr/educare

educare@una.ac.cr

Particularmente, en el área de la enseñanza de la biología, se encuentran varios estudios que analizan los TPL (Correa y Valbuena Ussa, 2017), aunque, al igual que en otras disciplinas, su implementación ha seguido un camino controvertido (Baldaia dos Santos, 2006). Algunas investigaciones (Caamaño, 2002; Crujeiras Pérez y Jiménez Aleixandre, 2015); Fernández Marchesi, 2014; Tenreiro-Vieira y Marques Vieira, 2006); dan cuenta de que las actividades experimentales implementadas en las aulas son muy pautadas, con protocolos tipo "receta de cocina", donde las oportunidades de aprendizaje para el estudiantado son escasas, ya que además de estar completamente estructurado su desarrollo, se le otorga poca o nula importancia a la interpretación de los resultados. Otro de los aspectos cuestionados en relación con los TPL es su idoneidad para el aprendizaje de conceptos, aunque se destaca su utilidad para el aprendizaje de los procedimientos científicos (López Rua y Tamayo Alzate, 2012).

Estas dificultades plantean la necesidad de reflexionar sobre el accionar docente vinculado a la realización de TPL y sus supuestos de base, pues al considerar que cuando los TPL no se limitan a la mera verificación de contenidos teóricos y se proponen actividades en las que el estudiantado interactúa con los contenidos, se favorece la construcción de conocimientos (Zorrilla, 2019). Para esto se debe ahondar en el pensamiento del personal docente actual y futuro de ciencias naturales. Un abordaje válido en tal sentido es a través de la identificación de sus representaciones sociales (RS), por cuanto posibilita el estudio de los fenómenos educativos en su complejidad desde un punto de vista psicosocial que permite conocer la forma en que los sujetos interpretan y construyen su conocimiento sobre la realidad (Jodelet, 2003).

La teoría de las RS otorga importancia tanto a los aspectos cognitivos, ya que supone sujetos activos, cuya actividad psicológica es guiada por procesos cognitivos, como también a los aspectos sociales de constitución de lo real, en cuanto que interviene, entre otras cuestiones, a través del contexto en el que se encuentran inmersos los grupos sociales y los individuos, la comunicación, la cultura, los códigos y los valores vinculados con sus pertenencias sociales específicas (Jodelet, 1986; Lombardo y Monchietti, 2015; Rodríguez Salazar, 2003). Moscovici $(1961,1979)$ define el concepto de RS como formas particulares de conocimiento, que permiten elaborar los comportamientos de los individuos, así como también permite su comunicación. Según señala Jodelet (1986), las RS son un tipo de conocimiento espontáneo, ingenuo, que "se constituye a partir de nuestras experiencias, pero también de las informaciones, conocimientos, y modelos de pensamiento que recibimos y transmitimos a través de la tradición, la educación y la comunicación social. De este modo, este conocimiento es en muchos aspectos, un conocimiento socialmente elaborado y compartido" (p. 473).

Por otra parte, la teoría del núcleo central (Abric, 2001) plantea que el núcleo de la representación se constituye como la parte más estable de esta, ya que es coherente, consensual y, además, está influido por la memoria colectiva del grupo y su sistema de valores. La estructura nuclear de una RS es la encargada de definir significados, brindar consistencia y permanencia a la representación, por lo que cualquier cambio en el núcleo resultará en una conversión de 
http://doi.org/10.15359/ree.25-3.9

la representación completa (Abric, 2001). De esta manera, según Petracci y Kornblit (2007), el núcleo cumple con dos funciones: (a) otorgar el significado a la representación y (b) organizar al resto de los elementos. Esta función de organización no resulta menor, ya que dos RS serán consideradas iguales si están organizadas alrededor del mismo núcleo, aunque el resto de su contenido sea diferente. De la misma forma, dos RS pueden ser distintas, aún cuando tengan el mismo contenido, si este está organizado con una estructura diferente. Por otra parte, la estructura también está conformada por un sistema periférico, cuyos elementos son más flexibles que los que integran el núcleo, permitiendo la adaptación de las RS a las características cambiantes del contexto y protegiendo al núcleo de posibles transformaciones (Abric, 2001). El sistema periférico permite el anclaje de la representación en el contexto concreto, adaptándola a nuevas informaciones que pudieran llegar a surgir de este. Flament (2001) asigna la función de prescribir comportamientos a los elementos periféricos, llevando al individuo a actuar espontáneamente en un contexto dado. Dichos elementos están jerarquizados, aquellos que se encuentren más cercanos al núcleo participarán en la concreción del significado de la representación, mientras que aquellos que se encuentran más alejados permiten ilustrar, aclarar o justificar esa significación (Abric, 2001).

Con base en lo expuesto, y como ya se adelantó, las RS se constituyen en un abordaje útil a la hora de analizar la práctica docente en relación con las actividades experimentales, ya que permiten detectar factores que obstaculizan y otros que benefician la enseñanza y el aprendizaje de las ciencias naturales, como se evidencia en muchas investigaciones en este contexto (Lacolla, 2012; Maturano, 2018; Mazzitelli, 2007; Mazzitelli, 2015; Zorrilla, 2019). Así, por ejemplo, un porcentaje relativamente alto de actitudes positivas relacionadas con el aprendizaje de las ciencias naturales en contrapunto con la presencia de actitudes negativas asociadas a la enseñanza de estas ciencias puede indicar una falta de adecuación de los recursos de enseñanza a las necesidades estudiantiles; e indicar, también, un problema relacionado con la enseñanza como proceso antes que con el contenido de esta. Por otra parte, una valoración positiva del conocimiento disciplinar podría estar actuando como un elemento favorecedor del aprendizaje en ciencias naturales (Mazzitelli y Aparicio, 2009).

Encualquiercaso, debe tenerse en cuenta quelas RS no deben interpretarse comola realidad misma. La importancia de explicitarlas se basa en el hecho de poder confrontar al personal docente con este conocimiento y reflexionar sobre la influencia en sus comportamientos, para lograr una comprensión más acabada de los TPL en relación con la enseñanza y el aprendizaje de las ciencias como objetos de la representación.

\section{Metodología}

En este trabajo, se presenta un estudio desarrollado desde un enfoque cualitativo con estudiantes del Profesorado en Biología de la provincia de San Juan, Argentina. Se realizó un muestreo por conveniencia, atendiendo al criterio de saturación para cada uno de los años de 
http://doi.org/10.15359/ree.25-3.9

http://www.una.ac.cr/educare

educare@una.ac.cr

formación de la carrera. Así, la muestra quedó conformada por 111 estudiantes de 1 ro a $4^{\text {to }}$ año. El estudio se llevó adelante con el objetivo de conocer las opiniones del estudiantado e identificar sus RS sobre los TPL para analizar de qué forma podrían incidir en su actual proceso de formación y en su futuro desempeño docente, e inferir posibles aspectos a tener en cuenta en relación con la realización de los TPL. Se implementó un instrumento que incluyó una técnica de evocación y jerarquización y una técnica de frases incompletas, que a continuación se detallan.

\section{Técnica de evocación y jerarquización:}

La técnica de evocación y jerarquización resulta una manera eficaz de acceder a la estructura y al contenido de las RS. Esto se debe a que permite identificar el núcleo central y los elementos periféricos que conforman la estructura de la representación. Esta es una técnica asociativa (Abric, 2001), a partir de un término inductor propuesto, los sujetos mencionan una serie de palabras o frases breves que relacionan con este. En un paso posterior, se solicita una jerarquización por orden de importancia de los elementos mencionados.

Petracci y Kornblit (2007) señalan que, al utilizar estas técnicas, los sujetos mencionan entre tres y cinco palabras espontáneamente, y que además se vuelve necesario incorporar una pregunta acerca del significado atribuido a cada palabra.

Así, para este estudio, y tomando como base investigaciones previas (Zorrilla, 2019), se propuso como término inductor la expresión prácticas de laboratorio y se pidió mencionar 5 palabras asociadas a este. Tomando como referencia investigaciones previas en esta misma temática (Zorrilla, 2019; Zorrilla y Mazzitelli, 2017), cabe aclarar que, aunque en las investigaciones actuales para referirse a las actividades experimentales se utiliza la expresión trabajos prácticos de laboratorio, en el instrumento implementado en este estudio se propuso la expresión prácticas de laboratorio, por ser la denominación de uso más habitual entre el estudiantado y el personal docente. A continuación, y con el fin de conocer la significación de cada uno de los términos anteriormente mencionados, se les pidió que fundamentaran las razones por las cuales habían elegido esas palabras, para de esta manera conocer las vinculaciones establecidas por los sujetos entre el término inductor y las palabras mencionadas.

Una vez concluido el proceso de recolección de las expresiones asociadas al término inductor prácticas de laboratorio y dada la gran cantidad de palabras que se mencionan, al igual que las múltiples significaciones que pueden tomar, se elaboraron categorías, a fin de agrupar las expresiones. Para construir dichas categorías se consideró además de los términos, la significación otorgada a dichas palabras por los sujetos. Asimismo, se realizó triangulación entre investigadores con el fin de considerar las opiniones de especialistas en el área de enseñanza de las ciencias naturales para la inclusión de las palabras en cada una de las categorías. Las categorías resultantes de este proceso pueden observarse en la Tabla 1: 
http://doi.org/10.15359/ree.25-3.9

Tabla 1: Categorías resultantes del procesamiento de la técnica de frases incompletas

\begin{tabular}{lll}
\hline \multicolumn{1}{c}{ Categoría } & \multicolumn{1}{c}{ Descripción } & \multicolumn{1}{c}{ Ejemplos } \\
\hline Actitudes y características & $\begin{array}{l}\text { Agrupa las expresiones que señalan } \\
\text { características relacionadas con el desarrollo de } \\
\text { los TPL, así como también las actitudes asociadas. }\end{array}$ & $\begin{array}{l}\text { Orden, divertidas, } \\
\text { compañerismo. }\end{array}$ \\
Conocimiento científico & $\begin{array}{l}\text { Incluye palabras que se vinculan con el } \\
\text { conocimiento de las ciencias naturales y, } \\
\text { particularmente, de las prácticas de laboratorio }\end{array}$ & $\begin{array}{l}\text { Física, membrana plasmática, } \\
\text { sustancia. }\end{array}$ \\
Enseñanza y aprendizaje & $\begin{array}{l}\text { Contiene las palabras relacionadas con los } \\
\text { procesos de enseñanza y de aprendizaje. }\end{array}$ & $\begin{array}{l}\text { Profesor, práctica, estudio. } \\
\text { Materiales y equipamiento } \\
\text { de laboratorio }\end{array}$ \\
$\begin{array}{l}\text { Se refiere a los elementos que se utilizan para el } \\
\text { desarrollo de las prácticas de laboratorio }\end{array}$ & $\begin{array}{l}\text { Microscopio, tubo de ensayo, } \\
\text { erlenmeyer. }\end{array}$ \\
Procesos y procedimientos & $\begin{array}{l}\text { Incluye palabras que se refieren a los } \\
\text { procedimientos propios de la construcción del } \\
\text { conocimiento científico. }\end{array}$ & $\begin{array}{l}\text { Observar, experimentación, } \\
\text { hipótesis. }\end{array}$ \\
\hline
\end{tabular}

Nota: Elaboración propia.

Posteriormente a la elaboración de categorías, se calculó la frecuencia de aparición de las palabras y el orden de importancia asignado por los sujetos, lo cual permite la identificación de la estructura de la representación (Graça et al., 2004; Mazzitelli, 2007; Petracci y Kornblit, 2007).

Siguiendo el proceso realizado por Mazzitelli $(2007,2015)$, para decidir cuándo considerar la frecuencia de aparición grande o pequeña, se analizaron las frecuencias de todas las categorías, por separado. Se calculó el promedio (p) entre la mayor y la menor frecuencia de las categorías. Luego, si n (frecuencia de aparición de una categoría) es mayor o igual a p, la frecuencia es grande y si n (frecuencia de aparición de una categoría) es menor que p, la frecuencia es pequeña.

El criterio utilizado para decidir cuándo la importancia asignada a cada categoría es alta o baja fue considerar para cada una de ellas los promedios, obtenidos de los valores de importancia asignados a cada una de las palabras que ingresaron a las respectivas categorías (entre 1 y 5). Así, la importancia será alta cuando el valor del promedio para la categoría sea menor que 3 y la importancia será baja cuando el promedio se encuentre entre 3 y 5 (Mazzitelli, 2007; Mazzitelli et al., 2009). 
http://doi.org/10.15359/ree.25-3.9

http://www.una.ac.cr/educare

educare@una.ac.cr

El análisis complementario de la frecuencia calculada y la importancia asignada permite decidir acerca de la centralidad de los términos mencionados. De esta manera, se procedió a agrupar las categorías en cuatro zonas (Mazzitelli, 2007) que admiten reconocer la estructura de las RS: Núcleo (frecuencia alta-importancia grande); primera periferia (frecuencia altaimportancia pequeña); segunda periferia (frecuencia baja-importancia pequeña) y elementos de contraste (frecuencia baja-importancia grande).

\section{Técnica de frases incompletas}

Sacks y Levy (1967) proponen la técnica de frases incompletas, en la cual se presenta una expresión que funciona como disparador para poder profundizar en diferentes opiniones e intereses de los sujetos. Dichas frases consisten en un conjunto de expresiones que el estudiantado, al completar, estructuran desde sus ideas, valores, creencias, dudas, etc.

Las frases incompletas propuestas, en este caso, se refieren a cómo fueron en el nivel secundario las prácticas de laboratorio y a cómo los sujetos preferían que fueran dichas prácticas. En todos los casos las expresiones usadas en esta técnica fueron construidas para que sus participantes pudieran expresar su opinión de manera abierta (Zorrilla y Mazzitelli, 2016), por lo cual se pidió incluir los detalles o las aclaraciones que consideraran convenientes para una mejor comprensión de sus opiniones.

Se tomaron como referencia investigaciones previamente realizadas por Zorrilla (2019) para estos procesos.

[Para] el procesamiento y análisis de las respuestas dadas por ... [los] estudiantes a la técnica de frases incompletas, se elaboraron categorías de análisis. ... Cabe destacar que las categorías construidas no son excluyentes, es decir que una misma respuesta puede incluirse en más de una categoría por las diferentes ideas expresadas, ya que se buscó captar toda la riqueza de las opiniones. Además, con el fin de reducir un posible sesgo tanto en la construcción de las categorías como en la agrupación de las respuestas, se realizó triangulación entre investigadores. Así, considerando las opiniones de especialistas en diversas disciplinas relacionadas con la enseñanza de las Ciencias Naturales, se ajustó el análisis para acordar la inclusión de las frases en las diferentes categorías. (p. 76)

Las categorías fueron elaboradas por separado para las frases "En la escuela secundaria las prácticas de laboratorio fueron... y En las prácticas de laboratorio prefiero..." (Zorrilla, 2019, p. 78); pero, dada la similitud en las respuestas, se unificaron. En la Tabla 2 se presenta las categorías resultantes. 
http://doi.org/10.15359/ree.25-3.9

Tabla 2: Categorías para la técnica de frases incompletas

\begin{tabular}{|c|c|}
\hline Categorías & Descripción \\
\hline Aspectos actitudinales (AA) & $\begin{array}{l}\text { Concentramos las expresiones referidas a las características que el } \\
\text { alumnado le atribuye a las prácticas de laboratorio y que genera } \\
\text { diferentes tipos de motivación para el aprendizaje. }\end{array}$ \\
\hline Cantidad o frecuencia (CyF) & $\begin{array}{l}\text { Agrupamos expresiones que hacen referencia a la cantidad o } \\
\text { frecuencia de prácticas realizadas. }\end{array}$ \\
\hline Caracterización de la modalidad de trabajo (MT) & $\begin{array}{l}\text { Agrupamos las expresiones relacionadas con la forma de } \\
\text { trabajar en las prácticas de laboratorio, teniendo en cuenta } \\
\text { la cantidad de personas, la finalidad de la práctica, los } \\
\text { diferentes momentos de trabajo (prelaboratorio, laboratorio } \\
\text { y poslaboratorio), las normas de seguridad, entre otras } \\
\text { características. }\end{array}$ \\
\hline Contribución de las prácticas al aprendizaje (A) & $\begin{array}{l}\text { Incluimos aquellas expresiones que mencionan las prácticas } \\
\text { de laboratorio en relación con situaciones de aprendizaje. }\end{array}$ \\
\hline Espacio y materiales (EyM) & $\begin{array}{l}\text { Incluimos las opiniones que hacen referencia al espacio } \\
\text { donde se realizan las prácticas y a los materiales de } \\
\text { laboratorio, como una parte en el proceso de construcción o } \\
\text { desarrollo de las prácticas. }\end{array}$ \\
\hline $\begin{array}{l}\text { Protagonismo en la diagramación o ejecución } \\
\text { de prácticas (DyE) }\end{array}$ & $\begin{array}{l}\text { Incluimos expresiones que hacen referencia al rol que } \\
\text { desempeña el estudiantado en las tareas de planificación y } \\
\text { ejecución de las actividades experimentales. }\end{array}$ \\
\hline Vinculación teoría-práctica (T-P) & $\begin{array}{l}\text { Agrupamos frases relacionadas con el tipo de vinculación } \\
\text { entre teoría y prácticas de laboratorio. }\end{array}$ \\
\hline
\end{tabular}

Nota: Elaboración con autorización y datos de Zorrilla (2019).

Una vez determinadas las categorías e incluidas las frases del estudiantado, se realizó el cálculo de la frecuencia de estas, con el objetivo de presentar los resultados en una gráfica apropiada para su análisis, para cada frase por separado, para visualizar de manera comparada la frecuencia de aparición de cada categoría.

\section{Análisis de resultados y discusión}

\section{Técnica de evocación y jerarquización}

A continuación, se presentan las estructuras de las RS identificadas, resultado del procesamiento de la técnica de evocación y jerarquización. Estas estructuras se presentan diferenciadas por el año de formación en la que se encuentra el estudiantado. 
http://doi.org/10.15359/ree.25-3.9

http://www.una.ac.cr/educare

educare@una.ac.cr

Tabla 3: Estructuras de las RS sobre prácticas de laboratorio del estudiantado del profesorado en biología, diferenciadas por año de formación

\begin{tabular}{|c|c|c|c|c|}
\hline & 1 er año & 2do año & 3er año & 4to año \\
\hline Núcleo & $\begin{array}{l}\text { - Materiales y } \\
\text { equipamiento de } \\
\text { laboratorio }\end{array}$ & $\begin{array}{l}\text { - Características y } \\
\text { actitudes }\end{array}$ & $\begin{array}{l}\text { - Características y } \\
\text { actitudes } \\
\text { - Enseñanza y } \\
\text { aprendizaje }\end{array}$ & $\begin{array}{c}\text { • Enseñanza y } \\
\text { aprendizaje }\end{array}$ \\
\hline Primera periferia & - & $\begin{array}{l}\text { - Materiales y } \\
\text { equipamiento de } \\
\text { laboratorio }\end{array}$ & $\begin{array}{l}\text { - Materiales y } \\
\text { equipamiento de } \\
\text { laboratorio }\end{array}$ & $\begin{array}{l}\text { - Procesos y } \\
\text { procedimientos }\end{array}$ \\
\hline Segunda periferia & $\begin{array}{l}\text { • Enseñanza } \\
\text { y aprendizaje }\end{array}$ & $\begin{array}{l}\text { - Procesos y } \\
\text { procedimientos } \\
\text { - Enseñanza y } \\
\text { aprendizaje }\end{array}$ & $\begin{array}{l}\text { - Conocimiento } \\
\text { científico } \\
\text { - Procesos y } \\
\text { procedimientos }\end{array}$ & $\begin{array}{l}\text { - Conocimiento } \\
\text { científico } \\
\text { - Materiales y } \\
\text { equipamiento de } \\
\text { laboratorio }\end{array}$ \\
\hline Zona de contraste & $\begin{array}{l}\text { - Conocimiento científico } \\
\text { - Procesos y } \\
\text { procedimientos } \\
\text { - Actitudes y } \\
\text { características }\end{array}$ & $\begin{array}{l}\text { - Conocimiento } \\
\text { científico }\end{array}$ & & \\
\hline
\end{tabular}

Nota: Elaboración propia.

Como puede observarse en la Tabla 3, la estructura de la RS predominante del estudiantado de $1^{\mathrm{er}}$ año se encuentra centrada en los materiales y el equipamiento necesario para llevar a cabo las prácticas de laboratorio; destaca la mención de palabras como microscopio y muestras, con altos niveles de importancia y frecuencia. Los elementos periféricos se encuentran únicamente en la segunda periferia y responden a la categoría enseñanza y aprendizaje, con un predominio de expresiones que relacionan los TPL como herramientas favorecedoras de un aprendizaje constructivo en el aula; destaca, entre las mismas, aprendizaje/aprender y prácticas. En este contexto solo se menciona como sujeto al profesor o profesora.

Por último, se destaca la cantidad de categorías que se encuentran en la zona de contraste, lo cual daría cuenta de la existencia de un grupo minoritario, en donde el núcleo de la RS que los caracteriza estaría compuesto por evocaciones que refieren a conceptos específicos y generales de biología, como microorganismos o célula, así como también por procesos, procedimientos y técnicas que se realizan en el marco de los TPL (técnicas, instructivo, observación). Además de esto, también se presentan evocaciones relacionadas con características y actitudes de estos, donde se priorizan las actitudes positivas como el compañerismo e interés. 
http://doi.org/10.15359/ree.25-3.9

En el caso del estudiantado de $2^{\text {do }}$ año, la estructura de su RS está centrada en evocaciones de la categoría características y actitudes. Dentro de la misma, se mencionan, en primer lugar, características relacionadas con la seguridad y la higiene en el laboratorio, tales como cuidado del material y precaución. En segundo lugar, se señalan actitudes asociadas a la responsabilidad durante el trabajo experimental (responsabilidad, estricto), así como un conjunto de actitudes positivas que relacionan los TPL con aspectos motivacionales (interés/interesantes, motivación).

En la primera periferia de esta RS, se encuentran las expresiones relacionadas con los materiales con los cuales se realizan los TPL. En general estas expresiones responden a materiales específicos del laboratorio, como cajas de Petri, microscopio y portaobjetos. Teniendo en cuenta la segunda periferia, se identifica en esta representación un componente procedimental, relacionado con la evocación de palabras principalmente vinculadas con los procesos que guiarían el desarrollo de un TPL, como observación y manipulación del material. También en esta segunda periferia se encuentra la categoría enseñanza y aprendizaje, cuyas evocaciones destacadas por importancia y frecuencia son objetivos, conocimientos previos y aprendizaje.

Por último, cabe señalar que al igual que ocurrió con el estudiantado de $1^{\text {er }}$ año, en la zona de contraste se observa la presencia de la estructura nuclear de un grupo minoritario que relaciona los TPL con elementos que pertenecen a conceptos específicos de biología (enzimas, membrana plasmática), por un lado, y a conceptos que refieren al entramado formal de las ciencias en general (teorías, conocimientos teóricos), por otro lado.

El núcleo de la RS del estudiantado de $3^{\text {er }}$ año presenta expresiones referidas a la categoría características y actitudes, al igual que de la categoría enseñanza y aprendizaje. Para la primera categoría, al igual que para el estudiantado de $2^{\text {do }}$ año, los elementos señalados presentan relación, por un lado, con las normas de seguridad en el laboratorio, donde destacan las palabras seguridad y precaución, las cuales evidenciarían actitudes positivas en relación con el trabajo en el laboratorio y con los cuidados necesarios a fin de evitar inconvenientes. Por otro lado, también se señalan características que implicarían el desarrollo de otras actitudes positivas, con mayor importancia y frecuencia las evocaciones: importante y curiosidad. Cabe mencionar que, a diferencia de las estructuras anteriores, aparecen también evocaciones relacionadas con la complejidad en el desarrollo de los TPL (difíciles y complejas). Además, la presencia de la categoría enseñanza y aprendizaje, con palabras como aprendizaje y construcción del conocimiento con mayores valores de importancia y frecuencia, daría cuenta de la valoración de los TPL como herramientas que favorecen la construcción del conocimiento en ciencias naturales.

En la primera periferia de esta representación se encuentran elementos de la categoría materiales y equipamiento de laboratorio, resaltando las evocaciones microscopio y material de laboratorio. La mayoría de las expresiones mencionadas son elementos específicos para la realización de TPL, lo que podría vincularse a una dependencia entre la realización de TPL y la disposición del material tradicional de laboratorio. Por último, la segunda periferia se encuentra compuesta por elementos de las categorías conocimiento científico y procesos y procedimientos. En el caso de 
http://doi.org/10.15359/ree.25-3.9

http://www.una.ac.cr/educare

educare@una.ac.cr

la primera, se mencionan conceptos generales como ciencia y conocimiento. En el segundo caso, se mencionan principalmente los pasos necesarios para llevar a cabo un TPL como observación y registro de datos, lo cual podría relacionarse con cierta sistematización en el desarrollo de los TPL.

Esta representación carece de zona de contraste, lo que permite inferir que existe una única RS para el estudiantado de $3^{\text {er }}$ año del profesorado de biología.

Para la estructura de la RS del estudiantado de $4^{\text {to }}$ año puede observarse que su núcleo está compuesto exclusivamente por expresiones referidas a la categoría enseñanza y aprendizaje, en lo cual destaca, por primera vez, en las representaciones analizadas, la mención de la palabra alumnos y la ausencia de la palabra profesor.

La primera periferia de esta representación está compuesta por palabras pertenecientes a la categoría procesos y procedimientos. La mayoría de estas palabras se refieren a los métodos y procedimientos, destacan por su importancia las palabras observación y comprobación. Teniendo en cuenta la segunda periferia de esta estructura, se encuentran evocaciones de la categoría conocimiento científico, que se refieren a contenidos generales de biología, destacándose célula por ser la palabra con mayor nivel de importancia y frecuencia. Además, también puede observarse la presencia de la categoría materiales y equipamiento de laboratorio, con menciones exclusivas a materiales de laboratorio. Se destacan, en este caso, las expresiones microscopio y preparados. Con respecto a esta categoría, cabe señalar que, a diferencia de las representaciones anteriores, en este caso la cantidad de evocaciones pertenecientes a esta categoría disminuye considerablemente. Esta situación podría dar cuenta de que, al finalizar la formación, se va produciendo una mayor independencia entre la realización de TPL y la disponibilidad de los materiales tradicionales con los cuales suelen realizarse.

Por último, cabe señalar que esta estructura carece de zona de contraste, por lo cual solo se identifica una RS, que es la que predomina.

Demanera general, puede señalarse en función delas estructuras previamente presentadas, que este estudiantado, de $1^{\text {ro }}$ a $4^{\text {to }}$ año, relaciona los TPL con la enseñanza y el aprendizaje, aunque con distintos niveles de valoración para los diferentes grupos. En los primeros años esta relación es más débil, conformando la zona periférica de la representación, pero en los últimos años estos elementos se presentan como constitutivos del núcleo. Este cambio podría deberse al proceso de formación docente.

Otro cambio que se observa es en relación con la categoría materiales y equipamiento de laboratorio. En el primer año parecería existir una dependencia entre la realización de las prácticas y la disponibilidad de los materiales tradicionales para la realización de estas, la que disminuye gradualmente a partir del segundo año. Esto podría significar que, a medida que avanzan en su formación, este estudiantado tiene la posibilidad de realizar prácticas más desligadas del laboratorio tradicional como espacio físico necesario para su realización, así como también de los materiales tradicionalmente usados en ellas. 
http://doi.org/10.15359/ree.25-3.9

En relación con los procesos y procedimientos asociados a los TPL, se observa que en un principio forman parte del núcleo únicamente de un grupo minoritario, pero esto se va modificando hasta llegar a la primera periferia en el último año de la formación docente. Así, resulta favorable que las cuestiones procedimentales asociadas al trabajo en el laboratorio puedan ser parte de la RS predominante.

Por último, un resultado que llama la atención es el lugar que presentan las evocaciones relacionadas con el conocimiento científico, ya que, en el mejor de los casos, se encuentran en la segunda periferia de la RS, esto muestra una desvinculación de los TPL en relación con el conocimiento conceptual.

\section{Técnica de frases incompletas}

A continuación, en la Figura 1, se muestra la distribución de las frecuencias porcentuales, discriminando los resultados de $1^{\text {ro }}$ a $4^{\text {to }}$ año, para las categorías elaboradas en el caso de la frase incompleta "En la escuela secundaria las prácticas de laboratorio fueron...."

Figura 1: Distribución de frecuencias porcentuales de las categorías para la frase En la escuela secundaria las prácticas de laboratorio fueron....

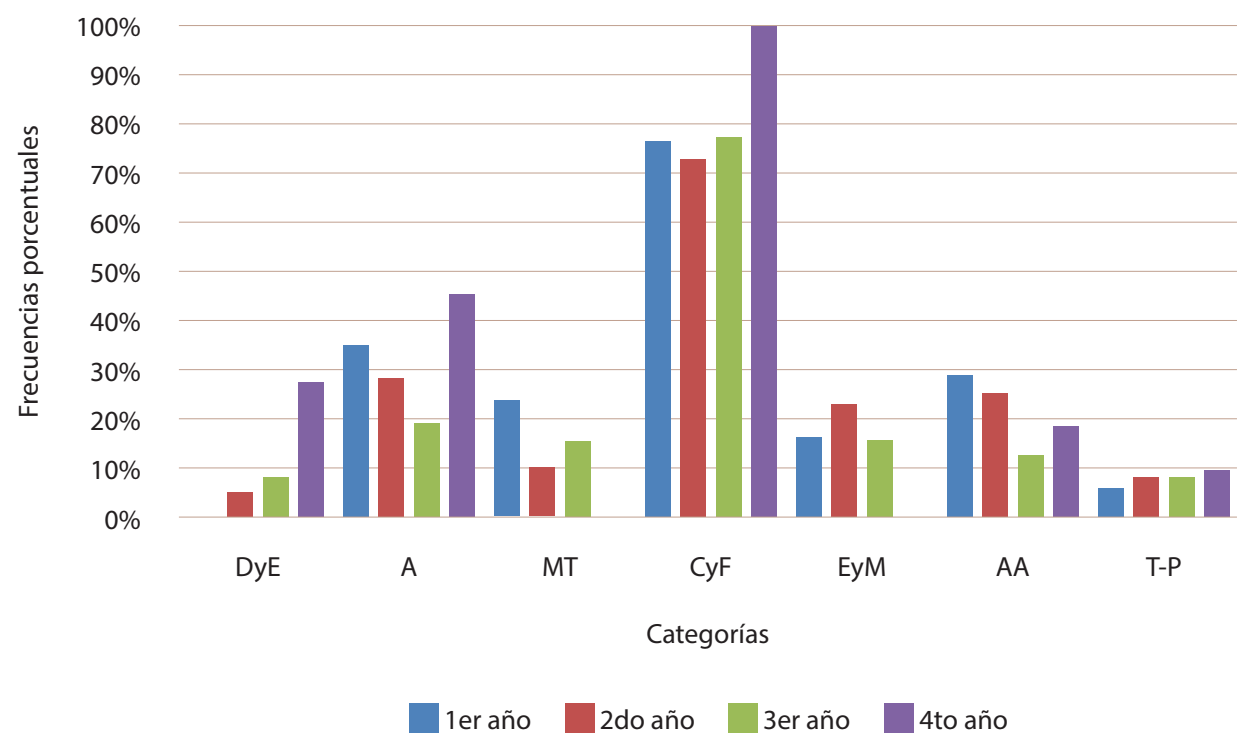

Nota: Elaboración propia. 
http://doi.org/10.15359/ree.25-3.9

http://www.una.ac.cr/educare

educare@una.ac.cr

Teniendo en cuenta las respuestas mencionadas por cada grupo de estudiantes aparece como rasgo común la escasa o nula realización de TPL, debido a que la categoría que mayor frecuencia presenta hace referencia a la cantidad o frecuencia de los TPL (CyF) y mencionan en todos los casos que el trabajo experimental en el nivel secundario es escaso o nulo. En los pocos casos que refieren haber realizado trabajo de laboratorio en la escuela secundaria, mencionan que el tiempo destinado fue breve.

Además, la escasez de trabajo experimental podría relacionarse tanto con la falta de un espacio adecuado para su realización como con la ausencia o el deterioro de material específico (EyM).

De manera general, también se destacan las respuestas vinculadas a la contribución de los TPL al aprendizaje (A). Los describen, principalmente, como favorecedores del aprendizaje. En algunos casos las respuestas son más una expresión de deseo que una descripción de una experiencia vivida, destacan que el aprendizaje hubiese sido más sencillo con la ayuda de TPL. Es poca la cantidad de estudiantes que señala que los TPL fueron de poca ayuda para el aprendizaje de los contenidos.

Disminuyendo la frecuencia porcentual, pero aún con una marcada presencia en las respuestas para los cuatro grupos de estudiantes, se destaca la categoría aspectos actitudinales (AA), donde principalmente se menciona que las prácticas fueron motivadoras, llamativas y divertidas. Esto permite inferir que los TPL desarrollados por el estudiantado de esta muestra habrían respondido a objetivos relacionados principalmente con aspectos actitudinales, con el fin de promover el gusto por las ciencias naturales en el alumnado

Por último, como puede observarse en la Figura 1, la categoría que presenta una escasa cantidad de respuestas de estudiantes de los cuatro años del Profesorado de Biología es la referida a la vinculación entre teoría y práctica (T-P). Estas respuestas se diferencian en dos grupos, donde en el primero se señala a los TPL como una instancia posterior y necesaria para la comprensión de los conocimientos teóricos, mientras que en el segundo grupo se menciona que los TPL no tenían relación con los temas teóricos analizados en clase.

En relación con las categorías restantes, se presentará a continuación el análisis de los aspectos más destacados:

- Los estudiantes de $2^{\text {do }}$ a $4^{\text {to }}$ año destacan mayor participación del profesorado que de estudiantes en la realización y ejecución de los TPL (DyE).

- Por otra parte, el espacio y los materiales involucrados en los TPL (EyM), al igual que la modalidad de trabajo (MT), solo son señalados por estudiantes de $1^{\text {ro }}, 2^{\text {do }}$ y $3^{\text {er }}$ año. 
http://doi.org/10.15359/ree.25-3.9

En el primer caso, tal cual fue adelantado previamente, las respuestas hacen referencia tanto a la falta de un espacio adecuado para la realización de los TPL como a la ausencia o el deterioro de material específico. En cuanto a la modalidad de trabajo, la mayoría de las respuestas señalan prácticas grupales y con falta de organización.

A continuación, en la Figura 2, se muestra la distribución de las frecuencias porcentuales para las categorías elaboradas para la frase incompleta "En las prácticas de laboratorio prefiero...."

Figura 2: Distribución de frecuencias porcentuales de las categorías para la frase En las prácticas de laboratorio prefiero....

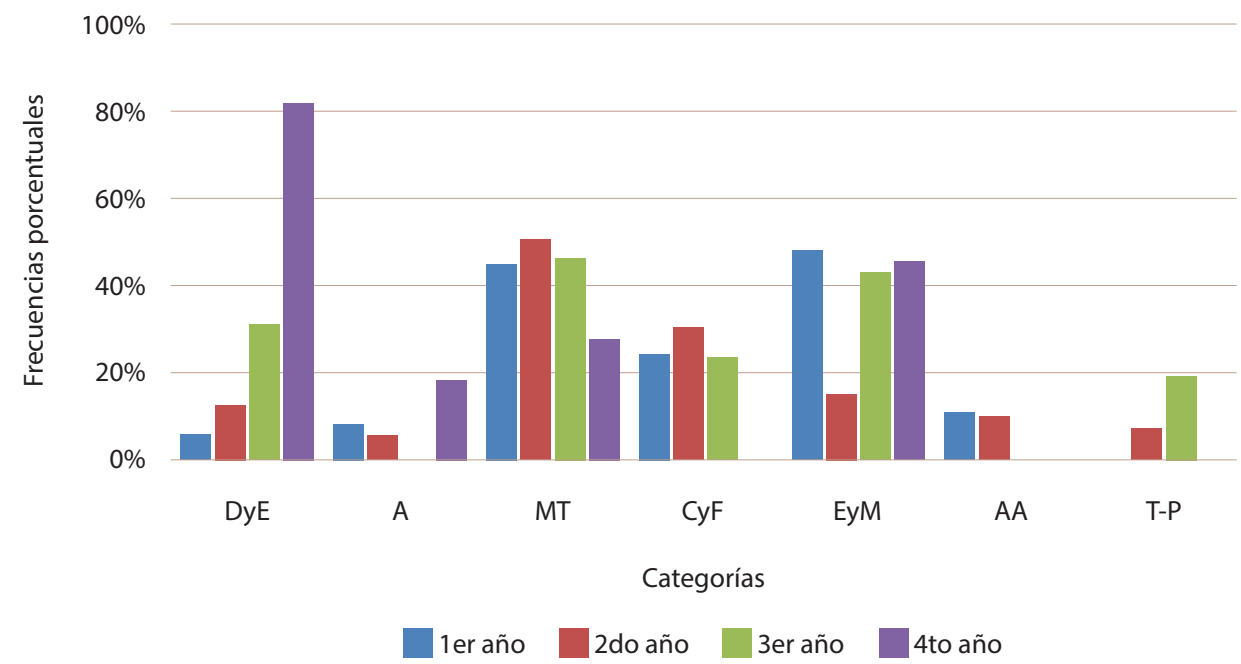

Nota: Elaboración propia.

De manera general, se destaca que, a pesar de las variaciones que se observan para cada grupo de estudiantes, pueden observarse algunas regularidades en las respuestas de sus estudiantes. Es así como, debido a la amplia presencia en los tres casos de la categoría caracterización de la modalidad de trabajo (MT), este grupo de estudiantes destaca su preferencia por el trabajo en el laboratorio en grupos con poca cantidad de integrantes, lo cual podría estar relacionado, principalmente, con la necesidad de un espacio físico adecuado para el desarrollo de las prácticas (lo que se ve reflejado en las expresiones de la categoría espacio y materiales).

Además de esto, el estudiantado de los tres primeros años expresa, entre sus principales preferencias, la realización de mayor cantidad y frecuencia de trabajos experimentales (CyF), en comparación con la realización actual de estos. Más allá de estas características principales, cabe destacar que también existen otros factores considerados entre sus preferencias, que varían según el año de formación. 
http://doi.org/10.15359/ree.25-3.9

http://www.una.ac.cr/educare

educare@una.ac.cr

En el caso del estudiantado de $4^{\text {to }}$ año, a diferencia del alumnado de los años anteriores, prefieren la realización de prácticas con mayor grado de apertura, reduciendo la participación del personal docente en ellas (DyE). Ello podría estar relacionado con la cercanía de este estudiantado a su futura práctica docente, lo que lo haría más reflexivo a la hora de considerar sus preferencias para la realización de TPL.

En relación con las categorías restantes, se puede observar, además de una escasa frecuencia de respuestas, que son mencionadas por poca cantidad de estudiantes. Así estudiantes de $1^{\text {ro }}, 2^{\text {do }}$ y $4^{\text {to }}$ año mencionan respuestas vinculadas a la contribución de las prácticas al aprendizaje (A), destacan entre sus preferencias que las prácticas sean beneficiosas para el aprendizaje. Así mismo, estudiantes de los dos primeros años mencionan características de los TPL que se relacionan con actitudes positivas (AA), como la preferencia por prácticas agradables y que generen asombro. Por último, estudiantes de $2^{\text {do }}$ y $3^{\text {er }}$ año mencionan la realización de TPL que contribuyan a entender o profundizar conocimientos teóricos analizados con anterioridad (T-P).

\section{Conclusiones}

Al considerar los resultados de las técnicas aplicadas, pueden identificarse algunos factores favorecedores, así como también otros que resultan obstaculizadores, para el aprendizaje de la biología en relación con los TPL.

En el caso de las estructuras de la RS sobre TPL que se encontraron para este alumnado, varían según el año de cursado, lo que evidencia cambios a lo largo de la formación docente, los cuales podrían estar vinculados a las características de los TPL realizados y al proceso mismo de formación. Dichos cambios resultan tendientes, por un lado, a centrar las representaciones en elementos de enseñanza y de aprendizaje y en aspectos procedimentales $y$, por el otro, descentrar la RS del equipamiento y el espacio físico tradicional en el cual suelen realizarse las prácticas.

Esta situación de descentración de las RS de los materiales y el equipamiento tradicional podría resultar beneficiosa, debido a que el estudiantado que se encuentra próximos a graduarse evidencia una posición más flexible que le permitirá adaptarse a las características de las instituciones en las que se inserte en su futura práctica docente, y desarrollar TPL con materiales no habituales y sin la necesidad de contar específicamente con un espacio convencional de laboratorio.

No obstante, se mantiene en todos los años, una escasa o nula vinculación de sus RS con los elementos relacionados con el conocimiento cientíico, lo cual podría generar, en la futura práctica docente, la realización de TPL que estén centrados en aspectos procedimentales o actitudinales, sin atender la vinculación con los aspectos conceptuales. 
http://doi.org/10.15359/ree.25-3.9

En cuanto a la técnica de frases incompletas referida a las prácticas de laboratorio durante el nivel secundario, puede destacarse que más allá de las diferencias señaladas, el factor común en estudiantes de los cuatro cursos es la escasa realización de prácticas experimentales, lo cual se constituye como un punto de partida para una futura reflexión acerca de la necesidad de incrementar el trabajo experimental en biología en el nivel secundario.

En relación con sus preferencias, el estudiantado menciona, con distinta frecuencia, según el año de cursado, el trabajo en grupos reducidos o individualmente y el protagonismo del alumnado durante la realización o diagramación de los TPL. Más allá de esto, es interesante destacar las respuestas donde el estudiantado señala que prefieren que los TPL tengan vinculación con conceptos teóricos estudiados, resultado que evidencia que esa falta de vinculación es detectada por el mismo estudiantado como una característica negativa que necesita ser atendida y superada.

\section{Referencias}

Abric, J. C. (2001). Prácticas sociales y representaciones. Ediciones Coyoacán.

Aguilar Muñoz, M., Fernández Tapia, M. y Durán Torres, C. (2011). Experiencias curiosas para enseñar química en el aula. Educación Química, (8), 23-34. https://bit.ly/368C4m5

Baldaia dos Santos, L. (2006). El cambio de las concepciones didácticas sobre las prácticas en la enseñanza de la biología. Alambique: Didáctica de las Ciencias Experimentales, (47), 23-29.

Bravo, A. A., Ramírez, G. P., Faúndez, C. A. y Astudillo, H. F. (2016). Propuesta didáctica constructivista para la adquisición de aprendizajes significativos de conceptos en física de fluidos. Formación universitaria, 9(2),105-114. http://dx.doi.org/10.4067/S0718-50062016000200012

Caamaño, A. (2002). ¿Cómo transformar los trabajos prácticos tradicionales en trabajos prácticos investigativos? Aula de Innovación Educativa, (113-114), 21-26. https://bit.ly/2JGhAtx

Carrascosa, J., Gil Pérez, D., Vilches, A. y Valdés, P. (2006). Papel de la actividad experimental en la educación científica. Caderno Brasileiro de Ensino de Física, 23(2),157-181. https:// periodicos.ufsc.br/index.php/fisica/article/view/6274/12764

Correa, M. A. y Valbuena Ussa, E. O. (2017). Estado del arte sobre los trabajos prácticos en la enseñanza de la biología abordados en publicaciones (2004-2006): Resultados relacionados con la imagen de práctica. Bio-grafía: Escritos sobre la biología y su enseñanza, 5(8), 117-135. https://doi.org/10.17227/20271034.8biografia117.135

Crujeiras Pérez, B., y Jiménez Aleixandre, M. P. (2015). Desafíos planteados por las actividades abiertas de indagación en el laboratorio: Articulación de conocimientos teóricos y prácticos en las prácticas científicas. Enseñanza de las ciencias: revista de investigación y experiencias didácticas, 33(1), 63-84. https://bit.ly/2I2F6AB 
http://doi.org/10.15359/ree.25-3.9

http://www.una.ac.cr/educare

educare@una.ac.cr

De Pro Bueno, A. (1998). ¿Se pueden enseñar contenidos procedimentales en las clases de ciencias? Enseñanza de las ciencias, 16(1), 21-41. https://ensciencias.uab.cat/article/view/ v16-n1-pro-bueno/2049

De Pro Bueno, A. (1999). Planificación de unidades didácticas por los profesores: Análisis de tipos de actividades de enseñanza. Enseñanza de las Ciencias, 17(3), 411-429. https:// ensciencias.uab.cat/article/view/v17-n3-pro-bueno/1977

Del Carmen, L. (2011). El lugar de los trabajos prácticos en la construcción del conocimiento científico en la enseñanza de la Biología y la Geología (pp. 91-106). En P. Cañal (Coord.), Didáctica de la biología y la geología. Grao.

Fernández Marchesi, N. E. (2014). Los trabajos prácticos de laboratorio de biología en los libros de texto de ciencias naturales para el nivel secundario utilizados en la Ciudad de Ushuaia [Tesis de maestría]. Universidad Nacional del Centro de la Provincia de Buenos Aires.

Fernández Marchesi, N. y Amórtegui, E. F. (2017). Trabajos de campo y de laboratorio: Dos escenarios en la enseñanza de la biología y la formación docente. Bio-grafía: Escritos sobre la biología y su enseñanza, (extraordinario), 1541-1547. https://doi.org/10.17227/biografia.extra2017-7335

Fernández Marchesi, N., Marcángeli, M. y Romero, C. (2011). Análisis de las estrategias de enseñanza de los docentes de ciencias naturales en dos escuelas públicas medias de tierra del fuego. Tecné, Episteme y Didaxis: TEA, (extraordinario), 1381-1386. https://www. researchgate.net/publication/258460101 Analisis de las estrategias de ensenanza de los docentes de ciencias naturales en dos escuelas publicas medias de tierra del_fuego

Flament, C. (2001). Estrutura e dinâmica das representações sociais. En D. Jodelet (Org.), As representações sociais (pp.173-186). Eduerj.

Gil, S. (1997). Nuevas tecnologías en la enseñanza de la física, oportunidades y desafíos. En Memorias 6 Conferencia Interamericana sobre Educación en la Física (pp. 13-15).

Graça, M. M., Moreira, M. A. y Caballero, C. (2004). Representacoes sobre a Matemática, seu ensino e aprendizagem: Um estudo exploratório. Investigações em Ensino de Ciências, 9(1), 37-93. https://bit.ly/36c0U4l

Hernández-Millán, G., Irazoque-Palazuelos, G. y López-Villa, N. M. (2012). ¿Cómo diversificar los trabajos prácticos? Un experimento ilustrativo y un ejercicio práctico como ejemplos. Educación Química, 23(1), 101-111. https://doi.org/10.1016/S0187-893X(17)30142-8

Hodson, D. (1994). Hacia un enfoque más crítico del trabajo de laboratorio. Enseñanza de las Ciencias, 12(3), 299-313. https://bit.ly/3mXbsv3 
http://doi.org/10.15359/ree.25-3.9

Jodelet, D. (1986). La representación social: Fenómenos, concepto y teoría. En S. Moscovici (Comp), Psicología social, II. Pensamiento y vida social. Psicología social y problemas sociales (pp. 469-494). Paidós.

Jodelet, D. (2003). Conferencia. En Primeras jornadas sobre representaciones sociales. CBC-UBA.

Lacolla, L. H. (2012). La representación social que los estudiantes poseen acerca de las reacciones químicas y su incidencia en la construcción del concepto de cambio químico [Tesis doctoral]. Universidad de Burgos. https://riubu.ubu.es/handle/10259/193

Lombardo, E. y Monchietti, A. (2015). Niveles de conocimiento que integran las representaciones sociales. Un estudio sobre polifasia cognitiva. Perspectivas en Psicología, 12(2), 50-56. https://bit.ly/38jlVwL

López Rua, A. M. y Tamayo Alzate, Ó. E. (2012). Las prácticas de laboratorio en la enseñanza de las ciencias naturales. Revista Latinoamericana de Estudios Educativos, 8(1), 145-166. https:// bit.ly/36abBoj

Maturano, C. (2018). El manual escolar en la enseñanza y el aprendizaje de las ciencias naturales: Análisis de representaciones sociales y aspectos didácticos de su utilización en la escuela secundaria [Tesis doctoral]. Universidad Nacional de Cuyo.

Mazzitelli, C. (2007). El aprendizaje de la física como reelaboración conceptual a la luz de algunas teorías psicosociales [Tesis doctoral]. Universidad Nacional de Cuyo.

Mazzitelli, C. (2015). La enseñanza de las ciencias y la formación docente inicial: Estudio de las representaciones sociales de estudiantes y docentes formadores. Editorial FFHA - UNSJ.

Mazzitelli, C. A. y Aparicio, M. T. (2009). Las actitudes de los alumnos hacia las ciencias naturales, en el marco de las representaciones sociales, y su influencia en el aprendizaje. Revista Electrónica de Enseñanza de las Ciencias, 8(1). https://dialnet.unirioja.es/servlet/ articulo?codigo $=3041509$

Mazzitelli, C., Aguilar, S., Guirado, A. M. y Olivera, A. (2009). Representaciones sociales de los profesores sobre la docencia: Contenido y estructura. Educación, Lenguaje y Sociedad, 6(6), 265-290. http://www.biblioteca.un/pam.edu.ar/pubpdf/ieles/n06a14mazzitelli.pdf

Merino, J. M. y Herrero F. (2007). Resolución de problemas experimentales de Química: Una alternativa a las prácticas tradicionales. Revista Electrónica de Enseñanza de las Ciencias, 6(3), 630-648. http://reec.uvigo.es/REEC/spanish/REEC_older_es.htm 
http://doi.org/10.15359/ree.25-3.9

http://www.una.ac.cr/educare

educare@una.ac.cr

Mordeglia, C., Cordero, S. y Dumrauf, A. G. (2006). Experimentando en ciencias naturales de tercer ciclo de EGB ¿Qué nos ofrecen los libros de texto? Memorias del $8^{\circ}$ Simposio de Investigadores en Enseñanza de la Física (pp. 220-228). https://www.academia. edu/5271289/EXPERIMENTANDO EN_CIENCIAS NATURALES_DE_TERCER_CICLO_DE EGB QU\%C3\%89 NOS OFRECEN LOS LIBROS DE TEXTO

Moscovici, S. (1961). La psychanalyse son image et son public: Étude sur la représentation sociale de la psychanalyse. Presses universitaires de France.

Moscovici, S. (1979). El psicoanálisis, su imagen y su público. Editorial Huemul.

Petracci, M. y Kornblit, A. (2007). Representaciones sociales: Una teoría metodológicamente pluralista. En A. L. Kornblit (Coord.), Metodologías cualitativas en ciencias sociales. Modelos y procedimientos de análisis (pp. 91-111). Biblos.

Rodríguez Salazar, T. (2003). El debate de las representaciones sociales en la psicología social. Relaciones: Estudios de historia y sociedad, 24(93), 52-80. https://bit.ly/38g2cOz

Sacks, J. y Levy, S. (1967). Test de frases incompletas. En L. Abt y L. Bellaw (Eds.). Psicología proyectiva. Enfoque clínico de la personalidad total (pp. 205-224). Paidós

Tenreiro-Vieira, C. y Marques Vieira, R. (2006). Diseño y validación de actividades de laboratorio para promover el pensamiento crítico de los alumnos. Revista Eureka sobre Enseñanza y Divulgación de las Ciencias, 3(3), 452-466. https://revistas.uca.es/index.php/eureka/article/ view/3850/3427

Zorrilla, E. G. (2019). Las prácticas de laboratorio en la enseñanza y el aprendizaje de las ciencias naturales desde una perspectiva psicosocial [Tesis doctoral]. Universidad Nacional de Cuyo. https://ri.conicet.gov.ar/bitstream/handle/11336/84028/CONICET_Digital_Nro.63bc84857a6f-4f5c-a570-3b93a83f0462 A.pdf?sequence $=2 \&$ isAllowed $=\mathrm{y}$

Zorrilla, E. G. y Mazzitelli, C. A. (2016). ¿Qué opinan los alumnos ingresantes a carreras de formación docente en ciencias naturales sobre las prácticas de laboratorio? Revista de Enseñanza de la Física, 28(extra), 77-83. https://revistas.unc.edu.ar/index.php/revistaEF/ article/view/15625/15432

Zorrilla, E. G. y Mazzitelli, C. A.(2017). Las representaciones sociales sobre las prácticas de laboratorio de estudiantes de los profesorados en biología, en física y en química. En L. M. Dubini, M. X. Erice, D. Meziat Luna, M. García Asete y L. Bengochea Martínez (Eds.), Educación científica e inclusión sociodigital (Vol. 3, pp. 1264-1270). Universidad de Alcalá, Servicio de Publicaciones. http://www.cieduc.org/2017/LibroCIEDUC2017-Volumen3.pdf 